\title{
A new application of Schrödinger-type identity to singular boundary value problem for the Schrödinger equation
}

Bo Meng ${ }^{1 *}$

\section{"Correspondence: \\ bmengust@hotmail.com \\ ${ }^{1}$ College of Mathematics and \\ Systems Science, Shandong \\ University of Science and}

Technology, Qingdao, China

\begin{abstract}
In this paper, we present a modified Schrödinger-type identity related to the Schrödinger-type boundary value problem with mixed boundary conditions and spatial heterogeneities. This identity can be regarded as an $L^{1}$-version of Fisher-Riesz's theorem and has a broad range of applications. Using it and fixed point theory in $L^{1}$-metric spaces, we prove that there exists a unique solution for the singular boundary value problem with mixed boundary conditions and spatial heterogeneities. We finally provide two examples, which show the effectiveness of the Schrödinger-type identity method.
\end{abstract}

Keywords: Boundary value problem; Schrödinger-type identity; Uniqueness

\section{Introduction}

In this paper, we consider a singular boundary value problem with mixed boundary conditions and spatial heterogeneities given by (see [1-7])

$$
\begin{aligned}
& -\Delta f=\chi f \quad \text { in }\rfloor, \\
& f=0 \quad \text { on }\urcorner_{1}, \\
& \left.\partial f+V(t) f=\chi \omega(t) u^{q} \quad \text { on }\right\rceil_{2}, q>1,
\end{aligned}
$$

where:

(i) $I=(0, W) \times(0, w)$ is a bounded rectangular domain, $I$ represents a porous medium with Lipschitz boundary $\left.\partial \beth=\rceil_{1} \cup\right\rceil_{2}$, where

$$
\urcorner_{2}=(\{0\} \times[0, w]) \cup([0, W] \times\{w\}) \cup(\{W\} \times[0, w])
$$

is the part in contact with air or covered by fluid, and

$$
\urcorner_{1}=[0, W] \times\{0\}
$$

is the impervious part of $\partial \beth$. Let $P=\beth \times(0, M)$, where $M>0$;

\section{Springer}


(ii) $-\Delta$ stands for the minus Laplacian operator, $\chi$ is a function of the variable $t$ satisfying

$$
c_{1} \leq \chi(t) \leq c_{2} \quad \text { for a.e. } t \in(0, W)
$$

for two positive constants $c_{1}$ and $c_{2}$, and $\omega(t)$ satisfies

$$
0 \leq \omega(t) \leq 1 \quad \text { for a.e. } t \in \mathrm{J} .
$$

(iii) the spatial heterogeneities on the boundary come given by the potentials

$\left.V, b \in \mathcal{C}(\rceil_{2}\right)$, where $b>0$ on $\rceil_{2}$, and $V$ possesses arbitrary sign at each point $\left.x \in\right\rceil_{2}$;

(iv) $\partial f(x)$ stands for the outer normal derivative of $f$ at $t \in\rceil_{2}$.

Our goal in this paper is to analyze the Schrödinger-type identity for (1.1). In the Schrödinger-type identity the continuous part of the corresponding Schrödinger operator is unchanged, and only the discrete part of the spectrum is changed by adding or removing a finite number of discrete eigenvalues to the spectrum. We can view the process of adding or removing discrete eigenvalues as changing the "unperturbed" potential and the "unperturbed" wavefunction into the "perturbed" potential and the "perturbed" wavefunction, respectively. Hence our goal is to present a Schrödinger-type identity at the potential and wavefunction levels by expressing the change in the potential and wavefunction in terms of quantities related to the perturbation and the unperturbed quantities.

The singular boundary value problem arises in many areas of applied mathematics and physics, and only its positive solution is significant in practice (see [8-12]). In recent years the study of positive solutions for ordinary elliptic systems and of positive radial solutions for elliptic systems in annular domains has received considerable attention; see [13-17] and the references therein. These references discussed mainly (1.1) for the particular case $\omega(t)=1$ and $V(t)=0$ and established some interesting results by applying the fixed point theorems of cone compression type, the lower and upper solutions method, and the fixed point index theory in cones, and especially extended the relevant results on the scalar second-order ordinary differential equations. For instance, Huang [18] has developed the Randon transform of the singular integral, where they have considered a linear stochastic Schrödinger equation in terms of local quantum Bernoulli noise. Subsequently, Sun [19] obtained new applications of the above identity for obtaining transmutations via the fixed point index for nonlinear integral equations. It is possible to derive a wide range of transmutation operators by this method. Zhang et al. [20] introduced a Schrödingertype identity for a Schrödinger free boundary problem in $\mathbb{R}^{n}$ and established necessary and sufficient conditions for the product of some distributional functions with uniformly sublinear term. Bahrouni et al. [21] obtained qualitative properties of entire solutions to a Schrödinger equation with sublinear nonlinearity and sign-changing potentials. Their analysis considered three distinct cases, and they established sufficient conditions for the existence of infinitely many solutions. In 2019, they [22] also considered the bound state solutions of sublinear Schrödinger equations with lack of compactness. Using variational methods, they proved the existence of two solutions with negative and positive energies, one of these solutions being nonnegative. Rybalko [23] studied an initial value problem for a one-dimensional nonstationary linear Schrödinger equation with a point singular 
potential. Xiang et al. [24] considered the existence and multiplicity of solutions for the Schrödinger-Kirchhof-type problems involving the fractional $p$-Laplacian and critical exponent. Xue and Tang [25] established the existence of bound state solutions for a class of quasilinear Schrödinger equations whose nonlinear term is asymptotically linear.

Recently, there have been also many extensive attentions (see [26, 27] and references therein) for singular Schrödinger-type boundary value problems under a general sublinear condition or a general superlinear condition involving the principal eigenvalue of the Schrödinger operator, and in some sense their conditions are optimal.

Our paper is organized as follows. In Sect. 2, we present a modified Schrödinger-type identity when a bound state is added to the spectrum of the Schrödinger operator. Applying it, in Sect. 3, we prove that there exists a unique solution for the singular boundary value problem with mixed boundary conditions and spatial heterogeneities. Finally, in Sect. 4, we present some illustrative examples for better understanding of the results introduced.

\section{A modified Schrödinger-type identity}

In this section, we introduce the following modified Schrödinger-type identity for the solution of (1.1). As for the classical Schrödinger-type identity, we refer the reader to [19] for more detail.

Lemma 2.1 Let

$$
\left.\left.\Sigma_{1}=\right\urcorner_{1} \times(0, M), \quad \Sigma_{2}=\right\urcorner_{2} \times(0, M), \quad \Sigma_{3}=\Sigma_{2} \cap\{\phi>0\}
$$

and

$$
\Sigma_{4}=\Sigma_{2} \cap\{\phi=0\} .
$$

(i) Let $\epsilon>0, k \geq 0$, and $\varsigma \in \mathfrak{D}\left(\mathbb{R}^{2} \times(0, M)\right)$ be such that $\varsigma \geq 0$ and $\varsigma=0$ on $\Sigma_{3}$. Then

$$
\int_{P} \chi(t)\left(f_{t}+\omega\right)\left(\min \left(\frac{(f-k)^{+}}{\epsilon}, \varsigma\right)\right)_{t} d t d s=0
$$

(ii) Let $\varsigma=0$ on $\Sigma_{2}$. Then

$$
\int_{P} \chi(t)\left(f_{t}+\omega\right)\left(\min \left(\frac{(k-u)^{+}}{\epsilon}, \varsigma\right)-\min \left(\frac{k}{\epsilon}, \varsigma\right)\right)_{t} d t d s=0
$$

Proof Let $\psi$ be a measure function satisfying

$$
d\left(\operatorname{supp}(\psi), \Sigma_{2}\right)>0
$$

and

$$
\operatorname{supp}(\psi) \subset \mathbb{R}^{2} \times(0, M) .
$$


Then we have that

$$
(t, s) \mapsto \pm \psi(t, t-\kappa)
$$

vanishes on $\Sigma_{2}$ and in $\Xi \times\{0, M\}$ for any $\kappa \in\left(-\kappa_{0}, \kappa_{0}\right)$, where $\kappa_{0}$ is a positive constant.

Note that there exist two constants $d_{1}>0$ and $d_{2}>0$ such that

$$
d_{1}\left\|f_{t}\right\|_{*} \leq\left\|f_{t}\right\|_{\mathcal{H}^{1}} \leq d_{2}\left\|f_{t}\right\|_{*} \quad \text { for all } f_{t} \in \Sigma_{1} .
$$

It follows from (1.2), (1.3), and (2.3) that

$$
\left\|\mathcal{F}_{l}\left(f_{t}\right)-\mathcal{F}_{l}\left(\bar{f}_{t}\right)\right\|_{*} \leq \frac{d_{2}}{d_{1}} \exp \left(\left(\frac{1}{2} R \tilde{K}_{R}-1\right) W\right)\left\|f_{t}-\bar{f}_{t}\right\|_{*}
$$

for all $f_{t}, \bar{f}_{t} \in \Sigma_{1}$.

So

$$
\begin{aligned}
& \left\|f_{l}(y)\right\|_{\mathcal{H}^{1}}^{2}+\left\|f_{l t}(y)\right\|_{\mathcal{H}^{1}}^{2}+2(1-\kappa) \int_{0}^{t}\left(\left\|f_{l}(y)\right\|_{\mathcal{H}^{1}}^{2}+\left\|f_{l t}(y)\right\|_{\mathcal{H}^{1}}^{2}\right) d y \\
& \leq \mathcal{R}^{2}+\frac{1}{2 \kappa} \int_{0}^{M}\|w(y)\|_{\mathcal{H}^{1}}^{2} d y \leq \mathcal{D}_{M} .
\end{aligned}
$$

On the other hand, we obtain that

$$
\begin{aligned}
& 2 \int_{0}^{M}\left\|f_{l}^{\prime}(y)\right\|_{\mathcal{H}^{1}}^{2} d y+\int_{0}^{M} \frac{d}{d y}\left[\left\|f_{l}(y)\right\|_{\mathcal{H}^{1}}^{2}+2 \int_{0}^{1} \tilde{V}\left(f_{l t}(t, y)\right) d x\right] d y \\
& \quad=2 \int_{0}^{M}\left\langle w(y), f_{l}^{\prime}(y)\right\rangle d y
\end{aligned}
$$

It follows from (2.5) and (2.6) that

$$
\begin{aligned}
& \int_{0}^{M} \frac{d}{d y}\left[\left\|f_{l}(y)\right\|_{\mathcal{H}^{1}}^{2}+2 \int_{0}^{1} \tilde{V}\left(f_{l t}(t, y)\right) d x\right] d y \\
& \quad=\left\|f_{l}(W)\right\|_{\mathcal{H}^{1}}^{2}-\left\|f_{l}(0)\right\|_{\mathcal{H}^{1}}^{2}+2 \int_{0}^{1}\left[\tilde{V}\left(f_{l t}(t, W)\right)-\tilde{V}\left(f_{l t}(t, 0)\right)\right] d x=0 .
\end{aligned}
$$

Putting $\iota_{R}=\sup _{|t| \leq \sqrt{2} R} \iota(t)$, we obtain that

$$
\begin{aligned}
& 2 \int_{0}^{M}\left\langle\iota\left(f_{l t}(y)\right) f_{l}(y), f_{l}^{\prime}(y)\right\rangle d y \\
& \quad \leq 2 \iota_{R} \int_{0}^{M}\left\|f_{l}(y)\right\|\left\|f_{l}^{\prime}(y)\right\| d y \\
& \quad \leq 2 R \iota_{R} \int_{0}^{M}\left\|f_{l}^{\prime}(y)\right\| d y \\
& \quad \leq 2 W \mathcal{R}^{2} \iota_{R}^{2}+\frac{1}{2} \int_{0}^{M}\left\|f_{l}^{\prime}(y)\right\|^{2} d y .
\end{aligned}
$$


From (2.6), (2.7), and (2.8) it follows that

$$
\int_{0}^{M}\left\|f_{l}^{\prime}(y)\right\|_{\mathcal{H}^{1}}^{2} d y \leq 2 W \mathcal{R}^{2} \iota_{R}^{2}+2 \int_{0}^{M}\|w(y)\|^{2} d y \leq \mathcal{D}_{W}
$$

for all $m \in \mathbb{N}$ and $t \in[0, M]$.

For fixed $i$ and $j$, from (2.9) we deduce that

$$
\begin{aligned}
& \int_{0}^{M}\left\langle f_{l}^{\prime}(y)+f_{l}(y), \chi_{j} \zeta_{i}(y)\right\rangle d y \rightarrow \int_{0}^{M}\left\langle f^{\prime}(y)+f(y), \chi_{j} \zeta_{i}(y)\right\rangle d y \\
& \int_{0}^{M}\left\langle f_{l t}^{\prime}(y)+f_{l t}(y), \chi_{j t} \zeta_{i}(y)\right\rangle d y \rightarrow \int_{0}^{M}\left\langle f_{t}^{\prime}(y)+f_{t}(y), \chi_{j t} \zeta_{i}(y)\right\rangle d y
\end{aligned}
$$

It follows from (2.8) that

$$
\begin{aligned}
& \int_{0}^{M}\left\langle\bar{V}\left(f_{l t}(y)\right), \chi_{j t} \zeta_{i}(y)\right\rangle d y \rightarrow \int_{0}^{M}\left\langle\bar{V}\left(f_{t}(y)\right), \chi_{j t} \zeta_{i}(y)\right\rangle d y, \\
& \int_{0}^{M}\left\langle\iota\left(f_{l t}(y)\right) f_{l}(y), \chi_{j} \zeta_{i}(y)\right\rangle d y \rightarrow \int_{0}^{M}\left\langle\iota\left(f_{t}(y)\right) f(y), \chi_{j} \zeta_{i}(y)\right\rangle d y .
\end{aligned}
$$

So

$$
\begin{aligned}
& \int_{0}^{M}\left\langle f^{\prime}(y)+f(y), \chi_{j} \zeta_{i}(y)\right\rangle d y+\int_{0}^{M}\left\langle f_{t}^{\prime}(y)+f_{t}(y), \chi_{j t} \zeta_{i}(y)\right\rangle d y \\
& \quad+\int_{0}^{M}\left\langle\bar{V}\left(f_{t}(y)\right), \chi_{j t} \zeta_{i}(y)\right\rangle d y+\int_{0}^{M}\left\langle\iota\left(f_{t}(y)\right) f(y), \chi_{j} \zeta_{i}(y)\right\rangle d y \\
& \quad=\int_{0}^{M}\left\langle w(y), \chi_{j} \zeta_{i}(y)\right\rangle d y,
\end{aligned}
$$

which yields the equation

$$
\begin{gathered}
\int_{0}^{M}\left\langle f^{\prime}(y)+f(y), \chi(y)\right\rangle d y+\int_{0}^{M}\left\langle f_{t}^{\prime}(y)+f_{t}(y), \chi_{t}(y)\right\rangle d y \\
\quad+\int_{0}^{M}\left\langle\bar{V}\left(f_{t}(y)\right), \chi_{t}(y)\right\rangle d y+\int_{0}^{M}\left\langle\iota\left(f_{t}(y)\right) f(y), \chi(y)\right\rangle d y \\
\quad=\int_{0}^{M}\langle w(y), \chi(y)\rangle d y \text { for all } w \in L^{2}\left(0, W ; \mathcal{H}_{0}^{1}\right) .
\end{gathered}
$$

It follows from (2.13) that modified Schrödinger-type identities (2.1) and (2.2) hold.

\section{Uniqueness of the solution}

In this section, we obtain our main result that a solution of problem (1.1) is unique. We assume that

$$
\chi \in C^{1}([0, W]) .
$$

Now we can state our uniqueness theorem. 
Theorem 3.1 The solution of problem (1.1) associated with the initial data $\omega_{0}$ is unique and satisfies

$$
f \in L^{\infty}\left(0, W ; \mathcal{H}_{0}^{1} \cap \mathcal{H}^{2}\right), \quad f^{\prime} \in L^{2}\left(0, W ; \mathcal{H}_{0}^{1}\right) .
$$

Furthermore, we have the estimate

$$
\|f\|_{L^{\infty}(\mathrm{I})} \leq \max \left\{\left\|\tilde{f}_{0}\right\|_{L^{\infty}\left(\Sigma_{2}\right)},\|f\|_{L^{\infty}(\mathrm{I})}\right\} .
$$

Proof Consider a special orthonormal basis $\left\{\chi_{j}\right\}$ on $\mathcal{H}_{0}^{1}: \chi_{j}(t)=\sqrt{2} \sin (j \pi x), j \in \mathbb{N}$,

$$
-\triangle \chi_{j}=\tau_{j} \chi_{j}, \quad \chi_{j} \in C^{\infty}([0,1]), \tau_{j}=(j \pi)^{2}, \quad j=1,2, \ldots
$$

Put (see [28])

$$
f_{l}(s)=\sum_{j=1}^{l} d_{l j}(s) \chi_{j}
$$

where

$$
\begin{aligned}
& \left\langle f_{l}^{\prime}(s), \chi_{j}\right\rangle+\left\langle f_{l t}^{\prime}(s), \chi_{j t}\right\rangle+\left\langle f_{l t}(s)+\bar{V}\left(f_{l t}(s)\right), \chi_{j t}\right\rangle \\
& \quad+\left\langle\left(1+\iota\left(f_{l t}(s)\right)\right) f_{l}(s), \chi_{j}\right\rangle=\left\langle w(s), \chi_{j}\right\rangle, \quad 1 \leq j \leq l, \\
& f_{l}(0)=f_{0 l},
\end{aligned}
$$

in which

$$
f_{0 l}=\sum_{j=1}^{l} \beta_{l j} \chi_{j} \rightarrow \tilde{f}_{0} \quad \text { strongly in } \mathcal{H}_{0}^{1} \cap \mathcal{H}^{2} .
$$

Equality (3.5) yields that

$$
\begin{aligned}
& d_{l i}^{\prime}(s)+d_{l i}(s)+\frac{1}{1+\tau_{i}}\left[\left\langle\bar{V}\left(f_{l t}(s)\right), \chi_{i x}\right\rangle+\left\langle l\left(f_{l t}(s)\right) f_{l}(s), \chi_{i}\right\rangle\right] \\
& \quad=\frac{1}{1+\tau_{i}}\left\langle w(s), \chi_{i}\right\rangle, \\
& d_{l i}(0)=\beta_{l i}, \quad 1 \leq i \leq l .
\end{aligned}
$$

Multiplying the $j$ th equation of (3.7) by $d_{l j}(s)$ and summing up with respect to $j$, we obtain that

$$
\mathcal{S}_{l}(s)=\mathcal{S}_{l}(0)+2 \int_{0}^{t}\left\langle w(y), f_{l}(y)\right\rangle d y
$$

where

$$
\begin{aligned}
\mathcal{S}_{l}(s)= & \left\|f_{l}(s)\right\|_{\mathcal{H}^{1}}^{2}+2 \int_{0}^{t}\left\|f_{l}(y)\right\|_{\mathcal{H}^{1}}^{2} d y \\
& +2 \int_{0}^{t}\left\langle\bar{V}\left(f_{l t}(y)\right), f_{l t}(y)\right\rangle d y+2 \int_{0}^{t}\left\langle\iota\left(f_{l t}(y)\right), f_{l}^{2}(y)\right\rangle d y .
\end{aligned}
$$


So

$$
\mathcal{S}_{l}(0)=\left\|f_{0 l}\right\|_{\mathcal{H}^{1}}^{2} \leq \bar{S}_{0}
$$

for $m \in \mathbb{N}$, where $f_{0 l} \rightarrow \tilde{f}_{0}$ strongly in $\mathcal{H}_{0}^{1} \cap \mathcal{H}^{2}$.

Consider

$$
y \bar{V}(y)=y \int_{0}^{y} V(r) d r \geq 0
$$

for $y \in \mathbb{R}$.

So

$$
\begin{aligned}
2 \int_{0}^{t}\left\langle w(y), f_{l}(y)\right\rangle d y & \leq \int_{0}^{t}\|w(y)\|^{2} d y+\int_{0}^{t}\left\|f_{l}(y)\right\|^{2} d y \\
& \leq \int_{0}^{W}\|w(y)\|^{2} d y+\frac{1}{2} \mathcal{S}_{l}(s),
\end{aligned}
$$

which yields that

$$
\mathcal{S}_{l}(s) \leq 2 \bar{S}_{0}+2 \int_{0}^{W}\|w(y)\|^{2} d y \leq D_{W}^{(1)} .
$$

Further, we obtain that

$$
\begin{aligned}
\left\langle f_{l t}^{\prime}(s), \chi_{j t}\right\rangle+\left\langle\Delta f_{l}^{\prime}(s), \Delta \chi_{j}\right\rangle+\left\langle\Delta f_{l}(s), \Delta \chi_{j}\right\rangle & \\
& +\left\langle f_{l t}(s), \chi_{j t}\right\rangle+\left\langle V\left(f_{l t}(s)\right) \Delta f_{l}(s), \Delta \chi_{j}\right\rangle \\
& +\left\langle\iota^{\prime}\left(f_{l t}(s)\right) f_{l}(s) \Delta f_{l}(s)+\iota\left(f_{l t}(s)\right) f_{l t}(s), \chi_{j t}\right\rangle \\
= & \left\langle f_{t}(s), \chi_{j t}\right\rangle, \quad 1 \leq j \leq l .
\end{aligned}
$$

Similarly,

$$
\begin{aligned}
\mathcal{P}_{l}(s)= & \mathcal{P}_{l}(0)-2 \int_{0}^{t}\left[\left\langle\iota^{\prime}\left(f_{l t}(y)\right) f_{l}(y) \Delta f_{l}(s), f_{l t}(y)\right\rangle\right. \\
& \left.+\left\langle\iota\left(f_{l t}(y)\right),\left|f_{l t}(y)\right|^{2}\right\rangle\right] d y+2 \int_{0}^{t}\left\langle f_{t}(y), f_{l t}(s)\right\rangle d y \\
= & \mathcal{P}_{l}(0)+\mathcal{I}_{1}+\mathcal{I}_{2},
\end{aligned}
$$

where

$$
\begin{aligned}
\mathcal{P}_{l}(s)= & \left\|f_{l t}(s)\right\|^{2}+\left\|\Delta f_{l}(s)\right\|^{2}+2 \int_{0}^{t}\left(\left\|f_{l t}(y)\right\|^{2}+\left\|\Delta f_{l}(y)\right\|^{2}\right) d y \\
& +2 \int_{0}^{t}\left\langle V\left(f_{l t}(y)\right),\left|\Delta f_{l}(s)\right|^{2}\right\rangle d y .
\end{aligned}
$$

On the other hand, we have

$$
\mathcal{P}_{l}(0)=\left\|f_{l t}(0)\right\|^{2}+\left\|\Delta f_{l}(0)\right\|^{2}=\left\|f_{0 m x}\right\|^{2}+\left\|\Delta f_{0 l}\right\|^{2} \leq \bar{P}_{0}
$$

for any $m \in \mathbb{N}$, where $\bar{P}_{0}$ always indicates a constant depending on $\tilde{f}_{0}$ (see [29]). 
It follows that

$$
\begin{aligned}
\mathcal{I}_{1}= & -2 \int_{0}^{t}\left[\left\langle\iota^{\prime}\left(f_{l t}(y)\right) f_{l}(y) \Delta f_{l}(s), f_{l t}(y)\right\rangle\right. \\
& \left.+\left\langle\iota\left(f_{l t}(y)\right),\left|f_{l t}(y)\right|^{2}\right\rangle\right] d y \leq 0
\end{aligned}
$$

and

$$
\begin{aligned}
\mathcal{I}_{2} & =2 \int_{0}^{t}\left\langle f_{t}(y), f_{l t}(s)\right\rangle d y \leq \int_{0}^{W}\left\|f_{t}(y)\right\|\left\|f_{l t}(y)\right\| d y \\
& \leq \int_{0}^{W}\left\|f_{t}(y)\right\| \sqrt{\mathcal{S}_{l}(y)} d y \leq \sqrt{D_{W}^{(1)}} \int_{0}^{W}\left\|f_{t}(y)\right\| d y
\end{aligned}
$$

from (3.14), (3.15), and (3.16).

So from (3.14), (3.16), (3.17), and (3.18) we have

$$
\mathcal{P}_{l}(s) \leq \bar{P}_{0}+\sqrt{D_{W}^{(1)}} \int_{0}^{W}\left\|f_{t}(y)\right\| d y \leq D_{W}^{(2)} .
$$

Define

$$
f_{l}(s)= \begin{cases}2\left(\frac{s}{\sigma}\right)^{2} & \text { if } s \in\left[0, \frac{\sigma}{2}\right] \\ 1-2\left(1-\frac{s}{\sigma}\right)^{2} & \text { if } s \in\left(\frac{\sigma}{2}, \sigma\right] \\ 1 & \text { if } s \in(\sigma, M-\sigma], \\ 1-2\left(1-\frac{M-t}{\sigma}\right)^{2} & \text { if } t \in\left(M-\sigma, M-\frac{\sigma}{2}\right], \\ 2\left(\frac{M-t}{\sigma}\right)^{2} & \text { if } t \in\left(M-\frac{\sigma}{2}, M\right],\end{cases}
$$

where $M \in(0, W]$, and $\sigma$ is a positive real number.

Note that $f_{l} \in C^{1}([0, W])$ and

$$
f_{l}^{\prime}(s)= \begin{cases}4 \frac{s}{\sigma^{2}} & \text { if } s \in\left[0, \frac{\sigma}{2}\right], \\ \frac{4}{\sigma}\left(1-\frac{s}{\sigma}\right) & \text { if } s \in\left(\frac{\sigma}{2}, \sigma\right], \\ 0 & \text { if } s \in(\sigma, M-\sigma], \\ -\frac{4}{\sigma}\left(1-\frac{M-s}{\sigma}\right) & \text { if } t \in\left(M-\sigma, M-\frac{\sigma}{2}\right] \\ -\frac{4}{\sigma}\left(\frac{M-s}{\sigma}\right) & \text { if } s \in\left(M-\frac{\sigma}{2}, M\right] .\end{cases}
$$

Let $\Sigma_{1}$ be the linear space generated by $\chi_{1}, \chi_{2}, \ldots, \chi_{l}$. We consider the following problem: Find a function $f_{l}(s)$ in the form (3.4) satisfying system (3.5) and the $W$-periodic condition (see [30])

$$
f_{l}(0)=f_{l}(W) .
$$

We consider the initial value problem given by (3.5), where $f_{0 l}$ is given in $\Sigma_{1}$. 
It follows that

$$
\begin{aligned}
& \frac{d}{d s}\left\|f_{l}(s)\right\|_{\mathcal{H}^{1}}^{2}+2\left\|f_{l}(s)\right\|_{\mathcal{H}^{1}}^{2}+2\left\langle\bar{V}\left(f_{l t}(s)\right), f_{l t}(s)\right\rangle \\
& \quad+2 \| \sqrt{\iota\left(f_{l t}(s)\right) f_{l}(s) \|^{2}} \\
& \quad=2\left\langle w(s), f_{l}(s)\right\rangle .
\end{aligned}
$$

So we have the following inequality:

$$
2\left\langle w(s), f_{l}(s)\right\rangle \leq \frac{1}{2 \delta_{1}}\|w(s)\|^{2}+2 \kappa\left\|f_{l}(s)\right\|^{2} \leq \frac{1}{2 \delta_{1}}\|w(s)\|^{2}+2 \kappa\left\|f_{l}(s)\right\|_{\mathcal{H}^{1}}^{2}
$$

for all $0<\delta_{1}<1$.

From (3.21) and (3.22) it follows that

$$
\begin{aligned}
& \frac{d}{d s}\left\|f_{l}(s)\right\|_{\mathcal{H}^{1}}^{2}+2\left(1-\delta_{1}\right)\left\|f_{l}(s)\right\|_{\mathcal{H}^{1}}^{2}+2\left\langle\bar{V}\left(f_{l t}(s)\right), f_{l t}(s)\right\rangle \\
& \quad+2 \| \sqrt{\iota\left(f_{l t}(s)\right) f_{l}(s) \|^{2}} \\
& \leq \frac{1}{2 \delta_{1}}\|w(s)\|^{2} .
\end{aligned}
$$

So

$$
\begin{aligned}
\frac{d}{d s} \| & f_{l t}(s)\left\|_{\mathcal{H}^{1}}^{2}+2\right\| f_{l t}(s)\left\|_{\mathcal{H}^{1}}^{2}+2\right\| \sqrt{V\left(f_{l t}(s)\right)} \Delta f_{l}(s) \|^{2} \\
& +2\left\langle\iota^{\prime}\left(f_{l t}(s)\right) f_{l}(s) \Delta f_{l}(s)+\iota\left(f_{l t}(s)\right) f_{l t}(s), f_{l t}(s)\right\rangle \\
= & 2\left\langle f_{t}(s), f_{l t}(s)\right\rangle .
\end{aligned}
$$

Similarly,

$$
\begin{aligned}
2\left\langle\iota^{\prime}\left(f_{l t}(s)\right) f_{l}(s) \Delta f_{l}(s)+\iota\left(f_{l t}(s)\right) f_{l t}(s), f_{l t}(s)\right\rangle & \\
= & 2 \int_{0}^{1} f_{l}(t, s) f_{l t}(t, s) \iota^{\prime}\left(f_{l t}(t, s)\right) \Delta f_{l}(x, s) d x \\
& +2 \int_{0}^{1} f_{l t}^{2}(t, s) \iota\left(f_{l t}(t, s)\right) d x \\
= & 2 \int_{0}^{1} f_{l}(t, s) \frac{\partial}{\partial t}\left(\int_{0}^{f_{l t}(t, s)} y \iota^{\prime}(y)\right) d x+2 \int_{0}^{1} f_{l t}^{2}(t, s) \iota\left(f_{l t}(t, s)\right) d x \\
= & -2 \int_{0}^{1} f_{l t}(t, s)\left(\int_{0}^{f_{l t}(t, s)} y \iota^{\prime}(y)\right) d x+2 \int_{0}^{1} f_{l t}^{2}(t, s) \iota\left(f_{l t}(t, s)\right) d x \\
= & 2 \int_{0}^{1}\left[f_{l t}^{2}(t, s) \iota\left(f_{l t}(t, s)\right)-f_{l t}(t, s)\left(\int_{0}^{f_{l t}(t, s)} y \iota^{\prime}(y)\right)\right] d x \geq 0,
\end{aligned}
$$


which implies

$$
\begin{aligned}
& \frac{d}{d s}\left\|f_{l t}(s)\right\|_{\mathcal{H}^{1}}^{2}+2\left(1-\delta_{1}\right)\left\|f_{l t}(s)\right\|_{\mathcal{H}^{1}}^{2}+2\left\|\sqrt{V\left(f_{l t}(s)\right)} \Delta f_{l}(s)\right\|^{2} \\
& \quad \leq \frac{1}{2 \delta_{1}}\left\|f_{t}(s)\right\|^{2} .
\end{aligned}
$$

From (3.23) and (3.26) it follows that

$$
\begin{aligned}
& \frac{d}{d s}\left[\left\|f_{l}(s)\right\|_{\mathcal{H}^{1}}^{2}+\left\|f_{l t}(s)\right\|_{\mathcal{H}^{1}}^{2}\right]+2\left(1-\delta_{1}\right)\left(\left\|f_{l}(s)\right\|_{\mathcal{H}^{1}}^{2}+\left\|f_{l t}(s)\right\|_{\mathcal{H}^{1}}^{2}\right) \\
& \quad \leq \frac{1}{2 \delta_{1}}\|w(s)\|_{\mathcal{H}^{1}}^{2},
\end{aligned}
$$

which, together with (3.27), gives

$$
\begin{aligned}
\| f_{l}(s) & \left\|_{\mathcal{H}^{1}}^{2}+\right\| f_{l t}(s) \|_{\mathcal{H}^{1}}^{2} \\
\leq & \left(\left\|f_{0 l}\right\|_{\mathcal{H}^{1}}^{2}+\left\|f_{0 m x}\right\|_{\mathcal{H}^{1}}^{2}-\mathcal{R}^{2}\right) e^{-2\left(1-\delta_{1}\right) t} \\
& +\left(\mathcal{R}^{2}+\frac{1}{2 \delta_{1}} \int_{0}^{t} e^{2\left(1-\delta_{1}\right) s}\|w(y)\|_{\mathcal{H}^{1}}^{2} d y\right) e^{-2\left(1-\delta_{1}\right) t} \\
\leq & \left(\left\|f_{0 l}\right\|_{\mathcal{H}^{1}}^{2}+\left\|f_{0 m x}\right\|_{\mathcal{H}^{1}}^{2}-\mathcal{R}^{2}\right) e^{-2\left(1-\delta_{1}\right) t}+\mathcal{R}^{2}
\end{aligned}
$$

where $\mathcal{R}^{2}=\sup _{0 \leq t \leq W} \mathcal{R}_{1}(s)$

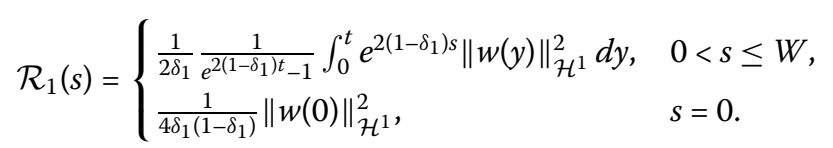

Note that $\left\|f_{0 l}\right\|_{\mathcal{H}^{1}}^{2}+\left\|f_{0 l x}\right\|_{\mathcal{H}^{1}}^{2} \leq \mathcal{R}^{2}$. It follows from (3.28) that

$$
\left\|f_{l}(s)\right\|_{\mathcal{H}^{1}}^{2}+\left\|f_{l t}(s)\right\|_{\mathcal{H}^{1}}^{2} \leq \mathcal{R}^{2}, \quad \text { that is, } \Sigma_{1}=W \text { for all } l .
$$

Let $\bar{B}_{l}(0, R)$ be a closed ball in the space $\Sigma_{1}$ of linear combinations of the functions $\chi_{1}$, $\chi_{2}, \ldots, \chi_{l}$. Put

$$
\begin{aligned}
& \mathcal{F}_{l}: \bar{B}_{l}(0, R) \rightarrow \bar{B}_{l}(0, R), \\
& f_{0 l} \mapsto f_{l}(W) .
\end{aligned}
$$

It is obvious that $y_{l}(s)$ satisfies

$$
\begin{aligned}
& \left\langle y_{l}^{\prime}(s)+y_{l}(s), \chi_{j}\right\rangle+\left\langle y_{l t}^{\prime}(s)+y_{l t}(s), \chi_{j t}\right\rangle \\
& \quad+\left\langle\bar{V}\left(f_{l t}(s)\right)-\bar{V}\left(\bar{f}_{l t}(s)\right), \chi_{j t}\right\rangle \\
& \quad+\left\langle\iota\left(f_{l t}(s)\right) f_{l}(s)-\iota\left(\bar{f}_{l t}(s)\right) \bar{f}_{l}(s), \chi_{j}\right\rangle=0 .
\end{aligned}
$$


Similarly,

$$
\begin{aligned}
& \frac{d}{d s}\left\|y_{l}(s)\right\|_{\mathcal{H}^{1}}^{2}+2\left\|y_{l}(s)\right\|_{\mathcal{H}^{1}}^{2}+2\left\langle\bar{V}\left(f_{l t}(s)\right)-\bar{V}\left(\bar{f}_{l t}(s)\right), y_{l t}(s)\right\rangle \\
& \left.\quad+2\left\langle\iota\left(f_{l t}(s)\right) f_{l}(s)-\iota\left(\bar{f}_{l t}(s)\right)\right) \bar{f}_{l}(s), y_{l}(s)\right\rangle=0 .
\end{aligned}
$$

From (3.33) it follows that

$$
\frac{d}{d s}\left\|y_{l}(s)\right\|_{\mathcal{H}^{1}}^{2}+\left(2-R \tilde{K}_{R}\right)\left\|y_{l}(s)\right\|_{\mathcal{H}^{1}}^{2} \leq 0
$$

which yields that

$$
\begin{aligned}
& \left\|y_{l}(W)\right\|_{\mathcal{H}^{1}}^{2} \leq e^{\left(R \tilde{K}_{R}-2\right) W}\left\|f_{0 l}-\bar{f}_{t}\right\|_{\mathcal{H}^{1}}^{2} \\
& \left\|\mathcal{F}_{l}\left(f_{0 l}\right)-\mathcal{F}_{l}\left(\bar{f}_{t}\right)\right\|_{\mathcal{H}^{1}} \leq \exp \left(\left(\frac{1}{2} R \tilde{K}_{R}-1\right) W\right)\left\|f_{0 l}-\bar{f}_{t}\right\|_{\mathcal{H}^{1}}
\end{aligned}
$$

We obtain that

$$
\begin{aligned}
\mathcal{Q}_{l}(s) & =\mathcal{Q}_{l}(0)-2 \int_{0}^{t}\left\langle\iota\left(f_{l t}(y)\right) f_{l}(y), f_{l}^{\prime}(y)\right\rangle d y+2 \int_{0}^{t}\left\langle w(y), f_{l}^{\prime}(y)\right\rangle d y \\
& =\mathcal{Q}_{l}(0)+\mathcal{J}_{1}+\mathcal{J}_{2}
\end{aligned}
$$

by multiplying the $j$ th equation of (3.35), where

$$
\begin{aligned}
& \mathcal{Q}_{l}(s)=\left\|f_{l}(s)\right\|_{\mathcal{H}^{1}}^{2}+2 \int_{0}^{t}\left\|f_{l}^{\prime}(y)\right\|_{\mathcal{H}^{1}}^{2} d y+2 \int_{0}^{1} \tilde{V}\left(f_{l t}(t, s)\right) d x, \\
& \tilde{V}(x)=\int_{0}^{z} \bar{V}(y) d y \geq 0 \quad \forall z \in \mathbb{R} .
\end{aligned}
$$

It is obvious that there exists a positive constant $\bar{Q}_{0}$ independent of $m$ such that

$$
\mathcal{Q}_{l}(0)=\left\|f_{0 l}\right\|_{\mathcal{H}^{1}}^{2}+2 \int_{0}^{1} \tilde{V}\left(f_{0 m x}(t)\right) d x \leq \bar{Q}_{0} \quad \forall m \in \mathbb{N}
$$

since $f_{0 l} \rightarrow \tilde{f}_{0}$ strongly in $\mathcal{H}_{0}^{1} \cap \mathcal{H}^{2}$.

From (3.19) it follows that

$$
\begin{aligned}
\left|f_{l t}(t, s)\right| & \leq\left\|f_{l t}(y)\right\|_{C^{0}([0,1])} \leq \sqrt{2}\left\|f_{l t}(y)\right\|_{\mathcal{H}^{1}} \\
& \leq \sqrt{2} \sqrt{\left\|f_{l t}(y)\right\|^{2}+\left\|\Delta f_{l}(y)\right\|^{2}} \leq \sqrt{2} \sqrt{2\left\|\Delta f_{l}(y)\right\|^{2}} \\
& \leq 2\left\|\Delta f_{l}(y)\right\| \leq 2 \sqrt{\mathcal{P}_{l}(y)} \leq 2 \sqrt{D_{W}^{(2)}},
\end{aligned}
$$


which yields that

$$
\begin{aligned}
\mathcal{J}_{1} & =-2 \int_{0}^{t}\left\langle\iota\left(f_{l t}(y)\right) f_{l}(y), f_{l}^{\prime}(y)\right\rangle d y \\
& \leq 2 \sup _{|x| \leq 2 \sqrt{D_{W}^{(2)}}} \iota(x) \int_{0}^{t}\left\|f_{l}(y)\right\|\left\|f_{l}^{\prime}(y)\right\| d y \\
& \leq 2 \sup _{|x| \leq 2 \sqrt{D_{W}^{(2)}}} \iota(x) \int_{0}^{t} \sqrt{\mathcal{S}_{l}(y)}\left\|f_{l}^{\prime}(y)\right\| d y \\
& \leq 2 \sqrt{D_{W}^{(1)}} \sup _{|x| \leq 2 \sqrt{D_{W}^{(2)}}} \iota(x) \int_{0}^{t}\left\|f_{l}^{\prime}(y)\right\| d y \\
& \leq 2 W D_{W}^{(1)} \sup _{|x| \leq 2 \sqrt{D_{W}^{(2)}}} \iota^{2}(x)+\frac{1}{2} \int_{0}^{t}\left\|f_{l}^{\prime}(y)\right\|^{2} d y \\
& \leq 2 W D_{W}^{(1)} \sup _{|x| \leq 2 \sqrt{D_{W}^{(2)}}} \iota^{2}(x)+\frac{1}{4} \mathcal{Q}_{l}(s)
\end{aligned}
$$

and

$$
\begin{aligned}
\mathcal{J}_{2} & =2 \int_{0}^{t}\left\langle w(y), f_{l}^{\prime}(y)\right\rangle d y \\
& \leq 2 \int_{0}^{W}\|w(y)\|^{2} d y+\frac{1}{2} \int_{0}^{t}\left\|f_{l}^{\prime}(y)\right\|^{2} d y \\
& \leq 2 \int_{0}^{W}\|w(y)\|^{2} d y+\frac{1}{4} \mathcal{Q}_{l}(s) .
\end{aligned}
$$

Combining (3.36) and (3.38)-(3.40), we have

$$
\mathcal{Q}_{l}(s) \leq 2\left(\bar{Q}_{0}+2 W D_{W}^{(1)} \sup _{|x| \leq 2 \sqrt{D_{W}^{(2)}}} \iota^{2}(x)+2 \int_{0}^{W}\|w(y)\|^{2} d y\right) \leq D_{W}^{(3)}
$$

It follows from (3.12), (3.19), and (3.41) that there exists a subsequence of $\left\{f_{l}\right\}$, still denoted by $\left\{f_{l}\right\}$, such that

$$
\begin{aligned}
& f_{l} \rightarrow u \quad \text { in } L^{\infty}\left(0, W ; \mathcal{H}_{0}^{1} \cap \mathcal{H}^{2}\right) \text { weakly*, } \\
& f_{l}^{\prime} \rightarrow f^{\prime} \quad \text { in } L^{2}\left(0, W ; \mathcal{H}_{0}^{1}\right) \text { weakly. }
\end{aligned}
$$

Applying the modified Schrödinger-type identity, by Lemma 2.1 there exists a subsequence of $\left\{f_{l}\right\}$ such that

$$
\begin{aligned}
& f_{l} \rightarrow f \quad \text { strongly in } L^{2}\left(0, W ; \mathcal{H}_{0}^{1}\right) \text { and a.e. in } \beth, \\
& f_{l t} \rightarrow f_{t} \quad \text { strongly in } L^{2}(\beth) \text { and a.e. in } \Xi .
\end{aligned}
$$


It follows from (3.43) that

$$
\begin{aligned}
& \left.\bar{V}\left(f_{l t}(t, s)\right) \rightarrow \bar{V}\left(f_{t}(t, s)\right) \quad \text { for a.e. }(t, s) \text { in }\right], \\
& \left.\iota\left(f_{l t}(t, s)\right) f_{l}(t, s) \rightarrow \iota\left(f_{t}(t, s)\right) f(t, s) \text { for a.e. }(t, s) \text { in }\right] .
\end{aligned}
$$

Inequalities (3.19) yield that

$$
\begin{gathered}
\left|f_{l t}(t, s)\right| \leq\left\|f_{l t}(s)\right\|_{C^{0}([0,1])} \leq \sqrt{2}\left\|f_{l t}(s)\right\|_{\mathcal{H}^{1}} \\
\leq 2\left\|\Delta f_{l}(s)\right\| \leq 2 \sqrt{\mathcal{P}_{l}(s)} \leq 2 \sqrt{D_{W}^{(2)}} ; \\
\left|\bar{V}\left(f_{l t}(t, s)\right)\right| \leq \sup _{|x| \leq 2 \sqrt{D_{W}^{(2)}}}|\bar{V}(x)| \leq D_{W} ; \\
\left|\iota\left(f_{l t}(t, s)\right) f_{l}(t, s)\right| \leq\left\|f_{l t}(s)\right\|\left|\iota\left(f_{l t}(t, s)\right)\right| \\
\leq \sqrt{D_{W}^{(2)}} \sup _{|x| \leq 2 \sqrt{D_{W}^{(2)}}}|\iota(x)| \leq D_{W} .
\end{gathered}
$$

It follows from (3.44) and (3.45) that

$$
\begin{aligned}
& \bar{V}\left(f_{l t}\right) \rightarrow \bar{V}\left(f_{t}\right) \quad \text { strongly in } L^{2}(\beth), \\
& \iota\left(f_{l t}\right) f_{l} \rightarrow \iota\left(f_{t}\right) u \quad \text { strongly in } L^{2}(\beth) .
\end{aligned}
$$

So

$$
\begin{aligned}
& \left\langle f_{s}(s), w\right\rangle+\left\langle f_{x s}(s), \chi_{t}\right\rangle+\left\langle f_{t}(s)+\bar{V}\left(f_{t}(s)\right), \chi_{t}\right\rangle+\left\langle\left(1+\iota\left(f_{t}(s)\right)\right) f(s), w\right\rangle \\
& \quad=\langle w(s), w\rangle, \quad \forall w \in \mathcal{H}_{0}^{1}, \\
& f(0)=\tilde{f}_{0} .
\end{aligned}
$$

Furthermore,

$$
f \in L^{\infty}\left(0, W ; \mathcal{H}_{0}^{1} \cap \mathcal{H}^{2}\right), \quad f^{\prime} \in L^{2}\left(0, W ; \mathcal{H}_{0}^{1}\right) .
$$

Let $f$ and $v$ be two weak solutions of (1.1) such that

$$
f, v \in L^{\infty}\left(0, W ; \mathcal{H}_{0}^{1} \cap \mathcal{H}^{2}\right), \quad f^{\prime}, v^{\prime} \in L^{2}\left(0, W ; \mathcal{H}_{0}^{1}\right)
$$

Put $\chi=f-v$, which satisfies

$$
\begin{aligned}
& \left\langle\chi_{s}(s), y\right\rangle+\left\langle\chi_{x s}(s), y_{t}\right\rangle+\left\langle\chi_{t}(s), y_{t}\right\rangle+\langle\chi(s), y\rangle \\
& \quad+\left\langle\bar{V}\left(f_{t}(s)\right)-\bar{V}\left(v_{t}(s)\right), y_{t}\right\rangle+\left\langle\iota\left(f_{t}(s)\right) u-\iota\left(v_{t}(s)\right) v, y\right\rangle=0, \quad \forall y \in \mathcal{H}_{0}^{1}, \\
& \chi(0)=0 \\
& u, v, \chi \in L^{\infty}\left(0, W ; \mathcal{H}_{0}^{1} \cap \mathcal{H}^{2}\right), \quad f_{s}, v_{s}, \chi_{s} \in L^{2}\left(0, W ; \mathcal{H}_{0}^{1}\right) .
\end{aligned}
$$


Define the following functions $\varrho_{1}$ and $\varrho_{2}$ of $\left(s_{1}^{1}, s_{1}^{2}\right)$ (resp. $\left.\left(s_{2}^{1}, s_{2}^{2}\right)\right)$ by

$$
\varrho_{1}\left(s_{1}\right)= \begin{cases}2\left(\frac{s_{1}-s_{1}^{1}}{\delta}\right)^{2} & \text { if } s_{1} \in\left[t_{1}^{1}, s_{1}^{1}+\frac{\delta}{2}\right], \\ 1-2\left(1-\frac{s_{1}-s_{1}^{1}}{\delta}\right)^{2} & \text { if } s_{1} \in\left(s_{1}^{1}+\frac{\delta}{2}, s_{1}^{1}+\delta\right], \\ 1 & \text { if } s_{1} \in\left(s_{1}^{1}+\delta, s_{1}^{2}-\delta\right], \\ 1-2\left(1-\frac{s_{1}^{2}-s_{1}}{\delta}\right)^{2} & \text { if } s_{1} \in\left(s_{1}^{2}-\delta, s_{1}^{2}-\frac{\delta}{2}\right], \\ 2\left(\frac{s_{1}^{2}-s_{1}}{\delta}\right)^{2} & \text { if } s_{1} \in\left(s_{1}^{2}-\frac{\delta}{2}, s_{1}^{2}\right],\end{cases}
$$

and

$$
\varrho_{2}\left(s_{2}\right)= \begin{cases}2\left(\frac{s_{2}-s_{2}^{1}}{\delta}\right)^{2} & \text { if } s_{2} \in\left[s_{2}^{1}, s_{2}^{1}+\frac{\delta}{2}\right], \\ 1-2\left(1-\frac{s_{2}-s_{2}^{1}}{\delta}\right)^{2} & \text { if } s_{2} \in\left(s_{2}^{1}+\frac{\delta}{2}, s_{2}^{1}+\delta\right], \\ 1 & \text { if } s_{2} \in\left(s_{2}^{1}+\delta, s_{2}^{2}-\delta\right], \\ 1-2\left(1-\frac{s_{2}^{2}-s_{2}}{\delta}\right)^{2} & \text { if } s_{2} \in\left(s_{2}^{2}-\delta, s_{2}^{2}-\frac{\delta}{2}\right], \\ 2\left(\frac{s_{2}^{2}-s_{2}}{\delta}\right)^{2} & \text { if } s_{2} \in\left(s_{2}^{2}-\frac{\delta}{2}, s_{2}^{2}\right] .\end{cases}
$$

Putting $y=\chi=u-v$ in (3.49) and integrating with respect to $t$, we have

$$
\begin{aligned}
\varrho(s)= & -2 \int_{0}^{t}\left\langle\bar{V}\left(f_{t}(y)\right)-\bar{V}\left(v_{t}(y)\right), \chi_{t}(y)\right\rangle d y \\
& -2 \int_{0}^{t}\left\langle\iota\left(f_{t}(y)\right) f(y)-\iota\left(v_{t}(y)\right) v(y), \chi(y)\right\rangle d y \\
= & \varrho_{1}(s)+\varrho_{2}(s),
\end{aligned}
$$

where

$$
\varrho(s)=\|\chi(s)\|_{\mathcal{H}^{1}}^{2}+2 \int_{0}^{t}\|\chi(y)\|_{\mathcal{H}^{1}}^{2} d y .
$$

Noting the monotonicity of the function $z \mapsto \bar{V}(x)$, we have

$$
\varrho_{1}(s)=-2 \int_{0}^{t}\left\langle\bar{V}\left(f_{t}(y)\right)-\bar{V}\left(v_{t}(y)\right), \chi_{t}(y)\right\rangle d y \leq 0 .
$$

Furthermore,

$$
\begin{aligned}
{\left[\iota\left(f_{t}\right) u-\iota\left(v_{t}\right) v\right] w } & =\left[\iota\left(f_{t}\right) w+\left(\iota\left(f_{t}\right)-\iota\left(v_{t}\right)\right) v\right] w \\
& =\iota\left(f_{t}\right) w^{2}+\left(\iota\left(f_{t}\right)-\iota\left(v_{t}\right)\right) v w \\
& \geq\left(\iota\left(f_{t}\right)-\iota\left(v_{t}\right)\right) v w
\end{aligned}
$$


which implies that

$$
\begin{aligned}
\varrho_{2}(s) & =-2 \int_{0}^{t}\left\langle\iota\left(f_{t}(y)\right) f(y)-\iota\left(v_{t}(y)\right) v(y), \chi(y)\right\rangle d y \\
& \leq-2 \int_{0}^{t}\left\langle\left[\iota\left(f_{t}(y)\right)-\iota\left(v_{t}(y)\right)\right] v(y), \chi(y)\right\rangle d y \\
& \leq 2 \int_{0}^{t}\left\|\left[\iota\left(f_{t}(y)\right)-\iota\left(v_{t}(y)\right)\right] v(y)\right\|\|\chi(y)\| d y \\
& \leq 2 \int_{0}^{t}\left\|\iota\left(f_{t}(y)\right)-\iota\left(v_{t}(y)\right)\right\|\left\|v_{t}(y)\right\|\|\chi(y)\| d y .
\end{aligned}
$$

Putting

$$
M=\|u\|_{L^{\infty}\left(0, W ; \mathcal{H}_{0}^{1} \cap \mathcal{H}^{2}\right)}+\|v\|_{L^{\infty}\left(0, W ; \mathcal{H}_{0}^{1} \cap \mathcal{H}^{2}\right)}
$$

and

$$
L_{M}=\sup _{|x| \leq M}\left|\iota^{\prime}(x)\right|
$$

we obtain that

$$
\left|\iota\left(f_{t}\right)-\iota\left(v_{t}\right)\right| \leq L_{M}\left|\chi_{t}\right| .
$$

So

$$
\begin{aligned}
\varrho_{2}(s) & \leq 2 L_{M} \int_{0}^{t}\left\|\chi_{t}(y)\right\|\left\|v_{t}(y)\right\|\|\chi(y)\| d y \\
& \leq 2 M L_{M} \int_{0}^{t}\left\|\chi_{t}(y)\right\|\|\chi(y)\| d y \\
& \leq M L_{M} \int_{0}^{t} \varrho(y) d y .
\end{aligned}
$$

Then from (3.50), (3.52), and (3.56) it follows that

$$
\varrho(s) \leq M L_{M} \int_{0}^{t} \varrho(y) d y,
$$

which leads to $\varrho(s)=0$, that is, $\chi=f-v=0$.

Let us assume that

$$
f_{0}(t) \leq M \quad \text { for a.e. } t \in \Sigma_{2} \quad \text { and } \quad \max \left\{\left\|\tilde{f}_{0}\right\|_{L^{\infty}},\|f\|_{L^{\infty}(\mathrm{I})}\right\} \leq M \text {. }
$$


Then $\omega=f-M$ satisfies

$$
\begin{aligned}
& \omega_{s}-\omega_{t t s}-\frac{\partial}{\partial t}\left(\omega_{t}+\bar{V}\left(x_{t}\right)\right)+z+(x+M) \iota\left(x_{t}\right) \\
& =w(t, s)-M, \quad 0<t<1,0<s<W, \\
& z(0, s)=z(1, s)=-M, \\
& z(t, 0)=\tilde{f}_{0}(t)-M .
\end{aligned}
$$

So

$$
\begin{gathered}
\left\langle\omega_{s}(s), v\right\rangle+\left\langle\omega_{x s}(s), v_{t}\right\rangle+\left\langle\omega_{t}(s)+\bar{V}\left(x_{t}(s)\right), v_{t}\right\rangle \\
+\left\langle z(s)+(x(s)+M) \iota\left(x_{t}(s)\right), v\right\rangle \\
=\langle w(s)-M, v\rangle \quad \text { for all } v \in \mathcal{H}_{0}^{1} .
\end{gathered}
$$

We deduce that the solution of the singular boundary value problem with mixed boundary conditions and spatial heterogeneities (1.1) satisfies $f \in L^{\infty}\left(0, W ; \mathcal{H}_{0}^{1} \cap \mathcal{H}^{2}\right), f^{\prime} \in$ $L^{2}\left(0, W ; \mathcal{H}_{0}^{1}\right)$, so that we are allowed to take $v=\omega^{+}=\frac{1}{2}(|x|+z)$ in $(3.60)$.

So

$$
\begin{aligned}
\left\langle\omega_{s}(s), \omega^{+}(s)\right\rangle+\left\langle\omega_{x s}(s), \omega_{t}^{+}(s)\right\rangle+\left\langle\omega_{t}(s)+\bar{V}\left(x_{t}(s)\right), \omega_{t}^{+}(s)\right\rangle & \\
& +\left\langle z(s)+(x(s)+M) \iota\left(x_{t}(s)\right), \omega^{+}(s)\right\rangle \\
= & \left\langle w(s)-M, \omega^{+}(s)\right\rangle,
\end{aligned}
$$

which yields that

$$
\begin{aligned}
\frac{1}{2} \frac{d}{d t}( & \left.\left\|\omega^{+}(s)\right\|^{2}+\left\|\omega_{t}^{+}(s)\right\|^{2}\right)+\left\|\omega_{t}^{+}(s)\right\|^{2}+\left\|\omega^{+}(s)\right\|^{2} \\
= & -\left\langle\bar{V}\left(x_{t}^{+}(s)\right), \omega_{t}^{+}(s)\right\rangle-\left\langle\left(x^{+}(s)+M\right) \iota\left(x_{t}^{+}(s)\right), \omega^{+}(s)\right\rangle \\
& +\left\langle w(s)-M, \omega^{+}(s)\right\rangle \leq 0
\end{aligned}
$$

and

$$
\begin{aligned}
\left\langle\omega_{s}(s), \omega^{+}(s)\right\rangle & =\int_{0}^{1} \omega_{s}(t, s) \omega^{+}(t, s) d x \\
& =\int_{0, z>0}^{1}\left(x^{+}(t, s)\right)_{t} \omega^{+}(t, s) d x \\
& =\frac{1}{2} \frac{d}{d t} \int_{0, z>0}^{1}\left|\omega^{+}(t, s)\right|^{2} d x \\
& =\frac{1}{2} \frac{d}{d t} \int_{0}^{1}\left|\omega^{+}(t, s)\right|^{2} d x \\
& =\frac{1}{2} \frac{d}{d t}\left\|\omega^{+}(s)\right\|^{2},
\end{aligned}
$$

and on the domain $z>0$, we have $\omega^{+}=z, \omega_{t}=\left(x^{+}\right)_{t}$, and $\omega_{s}=\left(x^{+}\right)_{s}$. 
It follows from (3.62) that

$$
\left\|\omega^{+}(s)\right\|^{2}+\left\|\omega_{t}^{+}(s)\right\|^{2} \leq\left\|\omega^{+}(0)\right\|^{2}+\left\|\omega_{t}^{+}(0)\right\|^{2}
$$

Since

$$
\begin{aligned}
& \omega^{+}(t, 0)=(f(t, 0)-M)^{+}=\left(\tilde{f}_{0}(t)-M\right)^{+}=0, \\
& \omega_{t}^{+}(t, 0)=0,
\end{aligned}
$$

we obtain that $\left\|\omega^{+}(s)\right\|^{2}+\left\|\omega_{t}^{+}(s)\right\|^{2}=0$. Thus $\omega^{+}=0$ and $f(t, s) \leq M$ for a.e. $(t, s) \in \beth$.

The case $-M \leq f_{0}(t)$ for a.e. $t \in \Sigma_{2}$ and

$$
M \geq \max \left\{\left\|\tilde{f}_{0}\right\|_{L^{\infty}},\|f\|_{L^{\infty}(\beth)}\right\}
$$

can be dealt with by considering $\omega=u+M$ and $\omega^{-}=\frac{1}{2}(|x|-z)$; we also have $\omega^{-}=0$, and hence $f(t, s) \geq-M$ for a.e. $(t, s) \in \beth$.

Furthermore, we obtain that $|f(t, s)| \leq M$ for a.e. $(t, s) \in \beth$, that is,

$$
\|u\|_{L^{\infty}(\boldsymbol{\Xi})} \leq M
$$

for all

$$
M \geq \max \left\{\left\|\tilde{f}_{0}\right\|_{L^{\infty}},\|f\|_{L^{\infty}(\beth)}\right\},
$$

which implies (3.3). The proof is complete.

\section{Examples}

In this section, we will test two singular boundary value problems with mixed boundary conditions and spatial heterogeneities by using the presented method.

Example 4.1 Consider the singular boundary value problem with mixed boundary conditions and spatial heterogeneities

$$
\begin{aligned}
& \left(4^{c} \mathcal{D}^{1 / 4}+3^{c} \mathcal{D}^{2 / 3}+2^{c} \mathcal{D}^{3 / 4}\right) s(y)=\frac{M}{\sqrt{t^{2}+81}}\left(\cos x+\cot ^{-1} t\right), \quad 0<y<1, \\
& s(0)=0, \quad s(1 / 4)=0, \quad s(1)=\int_{0}^{1 / 5} s(r) d r .
\end{aligned}
$$

Here $M>0$, and

$$
f(y, s)=\frac{M}{\sqrt{t^{2}+81}}\left(\cos x+\cot ^{-1} t\right) .
$$

Put $p_{0}=2$ and $p_{1}=p_{2}=3$. It is easy to see that they satisfy the conditions of Lemma 2.1 and

$$
|f(y, s)-f(y, t)| \leq \frac{2}{9} M|s-t|
$$


Using the given values, we know that $\phi \approx 0.44269$ and $\phi_{1} \approx 0.21725$. So

$$
|f(y, s)| \leq \frac{M(3+2 \pi)}{3 \sqrt{t^{3}+81}}=\theta(y)
$$

and $\ell \phi_{1}<1$ when $M<41.32901$.

On the one hand, all conditions of Theorem 3.1 hold. So problem (4.1)-(4.2) has at least one weak solution in $[0, W]$. On the other hand, $\ell \phi<1$ whenever $M<17.28439$. So it follows from Theorem 3.1 that there exists a unique weak solution for problem (4.1)-(4.2) in $[0,1]$.

Example 4.2 Consider the singular boundary value problem with mixed boundary conditions and spatial heterogeneities

$$
\begin{aligned}
& \left(3^{c} \mathcal{D}^{1 / 4}+3^{c} \mathcal{D}^{2 / 3}+2^{c} \mathcal{D}^{3 / 4}\right) s(y)=\frac{1}{4 \pi} \cos (2 \pi s)+\frac{|s|^{2}}{1+|s|^{2}}, \quad 0<y<1, \\
& s(0)=0, \quad s(1 / 4)=0, \quad s(1)=\int_{0}^{3 / 4} s(r) d r .
\end{aligned}
$$

Here

$$
f(y, s)=\frac{2}{3 \pi} \cos (3 \pi s)+\frac{|s|^{2}}{1+|s|^{2}} .
$$

Similarly to Example 4.1, we obtain that

$$
|f(y, s)| \leq\left|\frac{2}{3 \pi} \cos (3 \pi s)+\frac{|s|^{2}}{1+|s|^{2}}\right| \leq \frac{2}{3}\|s\|+3,
$$

$g(y)=1$, and $\psi(\|s\|)=\frac{1}{2}\|s\|+1$.

It is clear that $M>0.23971$ (we have used $\phi=0.38471$ ). Thus the conclusion of Theorem 3.1 applies to problem (4.3)-(4.4).

\section{Conclusions}

In this paper, we presented a modified Schrödinger-type identity related to the Schrödinger-type boundary value problem with mixed boundary conditions and spatial heterogeneities. This identity can be regarded as an $L^{1}$-version of Fisher-Riesz's theorem, and it had a broad range of applications. Using it and fixed point theory in $L^{1}$-metric spaces, we proved that there exists a unique solution for the singular boundary value problem with mixed boundary conditions and spatial heterogeneities. We finally provided two examples, which show the effectiveness of the Schrödinger-type identity method.

Acknowledgements

Not applicable.

Funding

This work was supported by the Post-Doctoral Applied Research Projects of Qingdao (no. 2015122) and the Scientific Research Foundation of Shandong University of Science and Technology for Recruited Talents (no. 2014RCJJ032). 
Availability of data and materials

Not applicable.

\section{Ethics approval and consent to participate}

Not applicable.

\section{Competing interests}

The author declares that he has no competing interests.

\section{Consent for publication}

Not applicable.

\section{Authors' contributions}

The author completed the paper and approved the final manuscript.

\section{Publisher's Note}

Springer Nature remains neutral with regard to jurisdictional claims in published maps and institutional affiliations.

Received: 23 July 2019 Accepted: 9 October 2019 Published online: 17 October 2019

\section{References}

1. Amann, H.: Nonlinear elliptic equations with nonlinear boundary conditions. In: Eckhaus, W. (ed.) New Developments in Differential Equations. Math Studies, vol. 21, pp. 43-63. North-Holland, Amsterdam (1976)

2. DiBenedetto, E., Friedman, A.: Periodic behaviour for the evolutionary dam problem and related free boundary problems. Commun. Partial Differ. Equ. 11(12), 1297-1377 (1986)

3. Mozer, J.: A new proof of De Giorgi's theorem concerning the regularity problem for elliptic differential equations Commun. Pure Appl. Math. 13(3), 457-468 (1960)

4. Huang, J., Zhang, Q.: Existence and multiplicity of solutions for a class of p-Laplacian equations in a bounded domain. J. Cent. China Norm. Univ. Nat. Sci. 52, 8-13 (2018)

5. Miller, K.S., Ross, B.: An Introduction to the Fractional Calculus and Fractional Differential Equations. Wiley, New York (1993)

6. Serrin, J.: Local behavior of solutions of quasilinear elliptic equations. Acta Math. 111, 247-302 (1964)

7. Rabinowitz, P.H.: Some global results for nonlinear eigenvalue problems. J. Funct. Anal. 7, 487-513 (1971)

8. Akahori, J., Ida, Y., Markowsky, G.: p-conformal maps on the triangular lattice. Stat. Probab. Lett. 151, 42-48 (2019)

9. Markowsky, G.: On the distribution of planar Brownian motion at stopping times. Ann. Acad. Sci. Fenn., Math. 43(2), 597-616 (2018)

10. Markowsky, G.: On the planar Brownian Green's function for stopping times. J. Math. Anal. Appl. 455(2), 1221-1233 (2017)

11. Qiao, L., Wu, Z., Lin, G.: Random attractor and Hausdorff dimension for the stochastic Cahn-Hilliard equation J. Yunnan Univ. Nat. Sci. 34(3), 249-257 (2012)

12. Markowsky, G.: A remark on the probabilistic solution of the Dirichlet problem for simply connected domains in the plane. J. Math. Anal. Appl. 464(2), 1143-1146 (2018)

13. Dai, M., Huang, Z., Xia, J., Meng, B., Wang, J., Shen, H.: Non-fragile extended dissipativity-based state feedback control for 2-D Markov jump delayed systems. Appl. Math. Comput. 362, 124571 (2019)

14. Hu, X., Xia, J., Wei, Y., Meng, B., Shen, H.: Passivity-based state synchronization for semi-Markov jump coupled chaotic neural networks with randomly occurring time delays. Appl. Math. Comput. 361, 32-41 (2019)

15. Meng, B., Wang, X.: Adaptive synchronization for uncertain delayed fractional-order Hopfield neural networks via fractional-order sliding mode control. Math. Probl. Eng. 2018, 1603629 (2018)

16. Wang, X., Wang, Z., Shen, H.: Dynamical analysis of a discrete-time SIS epidemic model on complex networks. Appl. Math. Lett. 94, 292-299 (2019)

17. Xing, M., Xia, J., Wang, J., Meng, B., Shen, $H_{.:}$Asynchronous $H_{\infty}$ filtering for nonlinear persistent dwell-time switched singular systems with measurement quantization. Appl. Math. Comput. 362, 124578 (2019)

18. Huang, A., Lin, L.: Finite dimensional approximation of linear stochastic Schrödinger equation in terms of localization of quantum Bernoulli noises. J. Shandong Univ. Nat. Sci. 52(12), 67-71 (2017)

19. Sun, D.: Schrödinger-type identity to the existence and uniqueness of a solution to the stationary Schrödinger equation. Bound. Value Probl. 2019,60 (2019)

20. Zhang, X., Liu, D., Yan, Z., Zhao, G., Yuan, Y.: Schrödinger-type identity for Schrödinger free boundary problems. Bound. Value Probl. 2018, 135 (2018)

21. Bahrouni, A., Ounaies, H., Rădulescu, V.D.: Infinitely many solutions for a class of sublinear Schrödinger equations with indefinite potentials. Proc. R. Soc. Edinb., Sect. A 145(3), 445-465 (2015)

22. Bahrouni, A., Ounaies, H., Rǎdulescu, V.D.: Bound state solutions of sublinear Schrödinger equations with lack of compactness. Rev. R. Acad. Cienc. Exactas Fís. Nat., Ser. A Mat. 113(2), 1191-1210 (2019)

23. Rybalko, Y.: Initial value problem for the time-dependent linear Schrödinger equation with a point singular potential by the unified transform method. Opusc. Math. 38(6), 883-898 (2018)

24. Xiang, M., Zhang, B., Rădulescu, V.D.: Superlinear Schrödinger-Kirchhoff type problems involving the fractional p-Laplacian and critical exponent. Adv. Nonlinear Anal. 9(1), 690-709 (2020)

25. Xue, Y., Tang, C.: Existence of a bound state solution for quasilinear Schrödinger equations. Adv. Nonlinear Anal. 8(1) 323-338 (2019)

26. Meng, B.: Existence and convergence results of meromorphic solutions to the equilibrium system with angular velocity. Bound. Value Probl. 2019, 88 (2019) 
27. Meng, B.: Minimal thinness with respect to the Schrödinger operator and its applications on singular Schrödinger-type boundary value problems. Bound. Value Probl. 2019, 91 (2019)

28. Gilardi, G.: A new approach to evolution free boundary problems. Commun. Partial Differ. Equ. 4, 1099-1123 (1979)

29. Rossi, J.D.: The blow-up rate for a semilinear parabolic equation with a nonlinear boundary condition. Acta Math. Univ. Comen. 67, 343-350 (1998)

30. Stein, E.M.: Singular Integrals and Differentiability Properties of Functions. Princeton University Press, Princeton (1970)

Submit your manuscript to a SpringerOpen ${ }^{\odot}$ journal and benefit from:

- Convenient online submission

Rigorous peer review

- Open access: articles freely available online

- High visibility within the field

- Retaining the copyright to your article

Submit your next manuscript at $\gg$ springeropen.com 